\title{
Poisson probability function can help optimize trauma operating room availability
}

\author{
David R. Sinclair, MD
}

Received: 8 November 2010/Accepted: 7 December 2010/Published online: 23 December 2010

(C) Canadian Anesthesiologists' Society 2010

\section{To the Editor,}

The Poisson probability distribution is a statistical function that describes the occurrence of random events. The probability of the event must be the same for any two intervals of equal length, and the occurrence or nonoccurrence of the event in any interval must be independent of the occurrence or non-occurrence in any other interval. ${ }^{1}$ The Poisson probability distribution is used often in industry to model random arrivals in waiting line situations. In the hospital setting, the Poisson distribution has been used to describe the arrival rate of patients to the intensive care unit. ${ }^{2,3}$ The Poisson probability function is defined by an equation that calculates the probability of a random event that follows a Poisson probability distribution (Table A). A simplified version is available using Excel® 2000 (Microsoft, Redmond, WA, USA) (Table B). Managers can use the probability function to guide the optimal supply of scarce resources to match a variable random demand.

The arrival of patients at a trauma centre is a random event that follows a Poisson probability distribution. The likelihood of patients requiring immediate surgery is similar in any two time periods of equal length, and the arrival or non-arrival of the patient is independent of the arrival or non-arrival in any other time period. The Poisson probability function can be used to calculate the probability of a patient arriving while all operating rooms are in use. Consider a Level 1 medical centre that has two trauma operating rooms. Equate the number of operating rooms to the number of patients arriving during the average operative time period, as this time interval represents the time

D. R. Sinclair, MD ( $ه)$

University of Miami, Miami, FL, USA

e-mail: dsinclair@med.miami.edu period in which each operating room would be occupied if both rooms were being utilized simultaneously. Comparing the number of patients arriving in the average operative time period according to the day of the week or the time of the year would account for weekday and seasonal variability. The probability that a third patient requiring emergency surgery could arrive at the trauma centre while the two operating rooms are occupied is 0.18 (Table C). Three patients from different accidents could also arrive at one time. The probability that three or more patients from independent accidents could arrive in a three-hour period is 0.323 (Table D). There is a $33 \%$ probability of needing additional operating room availability. It is conceivable that a single event causing multiple victims could occur, such as a major motor vehicle collision. The arrival of each patient at the trauma centre in any time interval would not be independent of the arrival of any other patient in the same time interval. Tracking of actual arrival rates for a specified period and analysis of correlation would help to address this arrival pattern.

Trauma centre medical directors can use the Poisson probability function to plan operating staffing and to estimate the likelihood of requiring additional operating room space. Important factors to consider include the number of operating rooms devoted to trauma surgery, the average length of cases in the trauma operating rooms, and the average number of patients who arrive at the trauma centre requiring immediate surgery. In an operating room with two dedicated trauma operating rooms, knowledge of a high probability of the arrival of a third trauma victim would guide management decisions. Operating room directors may choose to stagger cases in the non-trauma operating rooms to ensure that a room could be vacant in a short and predictable amount of time. Alternatively, one operating room could be booked with short cases of 
Table Poisson probability calculations

A. Poisson probability function: $\mathrm{f}(\mathrm{x})=\mu^{\mathrm{x}} \mathrm{e}^{-\mu} / \mathrm{x}$ !

where: $f(x)=$ the probability of $x$ events in an interval

$\mathrm{x}=$ number of events

$\mu=$ average number of events over a specified time interval $\mathrm{e}=2.71828$

B. $=$ POISSON $(\mathrm{x}, \mu$, cum $)$

where: $\mathrm{x}=$ number of events

$\mu=$ average (mean) number of events over a specified time interval

cum $=$ value reflecting exactly $\mathrm{x}(\mathrm{cum}=0)$ or the number of random events between zero and $\mathrm{x}$, inclusive $(\mathrm{cum}=1)$

C.

$\mathrm{x}=3^{\text {rd }}$ event

$\mu=2$ patient arrivals in a three-hour period

cum $=0$

probability $(3)=$ POISSON $(3,2,0)=0.180$

D.

$\mathrm{x}=3$ or more events

$\mu=2$ patient arrivals in a three-hour period

cum $=1$

probability $(\geq 3)=1-\operatorname{POISSON}(2,2,1)=0.323$ predictable duration. Knowledge of a high probability can also guide staffing plans to ensure optimal availability of the anesthesia care team.

Funding sources None.

Consultancies None.

Commercial or non-commercial affiliations None.

Competing interest None declared.

\section{References}

1. Anderson DR, Sweeney DJ, Williams TA. Statistics for Business and Economics, $11^{\text {th }}$ ed. Mason, $\mathrm{OH}$ : South-Western, Cengage Learning; 2010: 218-20.

2. Green $L V$. How many hospital beds? Inquiry $2002 ; 39: 400-12$.

3. Milne $E$, Whitty $P$. Calculation of the need for paediatric intensive care beds. Arch Dis Child 1995; 73: 505-7. 